\title{
Axion Kinetic Misalignment Mechanism
}

\author{
Raymond T. Co $\odot,{ }^{1}$ Lawrence J. Hall $\odot,{ }^{2,3}$ and Keisuke Harigaya $\odot^{4}$ \\ ${ }^{1}$ Leinweber Center for Theoretical Physics, University of Michigan, Ann Arbor, Michigan 48109, USA \\ ${ }^{2}$ Department of Physics, University of California, Berkeley, California 94720, USA \\ ${ }^{3}$ Theoretical Physics Group, Lawrence Berkeley National Laboratory, Berkeley, California 94720, USA \\ ${ }^{4}$ School of Natural Sciences, Institute for Advanced Study, Princeton, New Jersey 08540, USA
}

(Received 22 November 2019; revised manuscript received 6 April 2020; accepted 8 June 2020; published 26 June 2020)

\begin{abstract}
In the conventional misalignment mechanism, the axion field has a constant initial field value in the early Universe and later begins to oscillate. We present an alternative scenario where the axion field has a nonzero initial velocity, allowing an axion decay constant much below the conventional prediction from axion dark matter. This axion velocity can be generated from explicit breaking of the axion shift symmetry in the early Universe, which may occur as this symmetry is approximate.
\end{abstract}

DOI: 10.1103/PhysRevLett.124.251802

Introduction.-Why is $C P$ violation so suppressed in the strong interaction [1-3] while near maximal in the weak interaction? The Peccei-Quinn (PQ) mechanism [4,5] provides a simple and elegant answer: the angular parameter describing $C P$ violation in the strong interaction is actually a field resulting from spontaneous symmetry breaking, $\theta(x)$. A potential $V(\theta)$ arises from the strong interaction and has $C P$ conserving minima, as shown in Fig. 1. Axions are fluctuations in this field $[6,7]$ and the mass of the axion is powerfully constrained by particle and astrophysics, $m_{a}<60 \mathrm{meV}$; equivalently, there is a lower bound on the PQ symmetry breaking scale $f_{a}=10^{8} \mathrm{GeV}$ $\left(60 \mathrm{meV} / m_{a}\right)[8-14]$.

In the early Universe, if the initial value of the field, $\theta_{i}$, is away from the minima, the axion field starts to oscillate at a temperature $T_{*}$ when $m_{a} \sim 3 H$, where $H$ is the Hubble expansion rate. These oscillations, illustrated in the upper diagram of Fig. 1, can account for the observed dark matter [15-17]. For $\theta_{i}$ not accidentally close to the bottom nor the hilltop of the potential, this "misalignment" mechanism predicts an axion mass of order $10 \mu \mathrm{eV}$ and tends to underproduce for heavier masses.

In this Letter we show that an alternative initial condition for the axion field, $\dot{\theta} \neq 0$, leads to axion dark matter for larger values of $m_{a}$. This "kinetic misalignment" mechanism is operative if the axion kinetic energy is larger than the potential energy at temperature $T_{*}$, delaying the onset of axion field oscillations, as shown in the lower diagram of Fig. 1. We begin with an elaboration of the basic

Published by the American Physical Society under the terms of the Creative Commons Attribution 4.0 International license. Further distribution of this work must maintain attribution to the author(s) and the published article's title, journal citation, and DOI. Funded by SCOAP ${ }^{3}$. mechanism. We then show that a sufficient $\dot{\theta}$ can arise at early times from explicit breaking of the PQ symmetry by a higher dimensional operator in the same manner as the Affleck-Dine mechanism, which generates rotations of complex scalar fields $[18,19]$.

The PQ symmetry is an approximate symmetry which is explicitly broken by the strong interaction. It is plausible that higher dimensional operators also explicitly break the PQ symmetry. Although they should be negligible in the vacuum in order not to shift the axion minimum from the $C P$ conserving one, they can be effective in the early Universe if the PQ symmetry breaking field takes a large initial value. Higher dimensional PQ-breaking operators are in fact expected if one tries to understand the PQ symmetry as an accidental symmetry arising from some exact symmetries [20-23]. The kinetic misalignment mechanism is therefore a phenomenological prediction

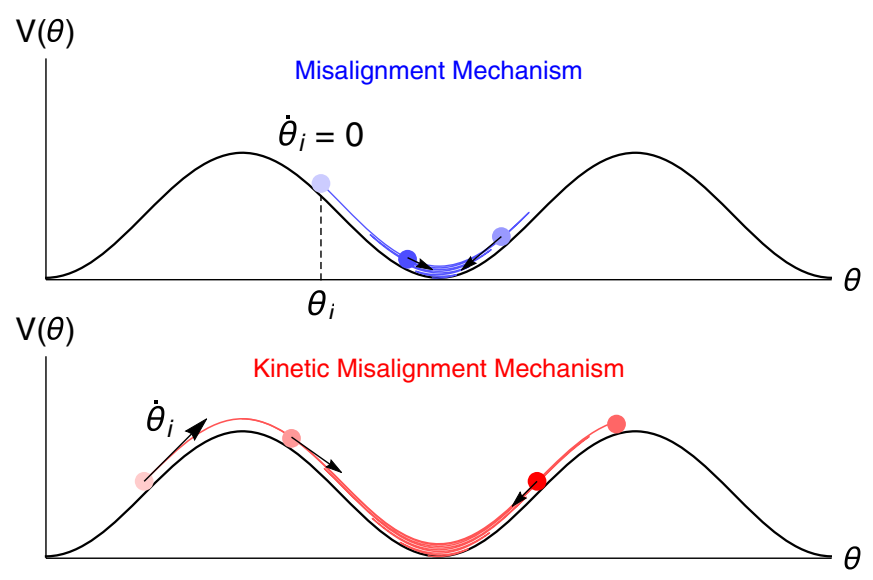

FIG. 1. The schematics of the (kinetic) misalignment mechanism. Initial conditions are labeled, shadings from light to dark indicate the time sequence of the motion, and arrows with different relative lengths denote instantaneous velocities. 
intrinsically tied to the theoretical origin of the $\mathrm{PQ}$ symmetry.

The mechanism allows for axion dark matter with a mass above the prediction of the standard misalignment mechanism. This mass scale $m_{a}=\mathcal{O}(0.1-100) \mathrm{meV}$ is under extensive experimental investigation [24-38]. Other known production mechanisms in this mass range are (1) parametric resonance from a PQ symmetry breaking field $[39,40]$, (2) anharmonicity effects [41-43] when $\theta_{i}$ approaches $\pi$ due to fine-tuning or inflationary dynamics $[44,45]$, (3) decays of unstable domain walls [46-53], and (4) production during a kination era [54]. Contrary to these mechanisms, kinetic misalignment offers an exciting theoretical connection with the baryon asymmetry of the Universe through so-called axiogenesis [55].

Kinetic misalignment mechanism.-We estimate the dark matter abundance for a generic axionlike field with decay constant $f_{\phi}, \phi=f_{\phi} \theta$, when $\dot{\theta} \neq 0$. Without loss of generality we take $\dot{\theta}>0$. It is convenient to express the rotation by the dimensionless quantity $\dot{\theta} f_{\phi}^{2} / s$ where $s \propto$ $R^{-3}$ is the entropy density and $R$ is the scale factor. In fact, $\dot{\theta} f_{\phi}^{2}$ is the Noether charge associated with the shift symmetry $\phi \rightarrow \phi+\alpha f_{\phi}$ and hence should decrease in proportion to $R^{-3}$. Therefore, $\dot{\theta} f_{\phi}^{2} / s$ remains constant. We define

$$
n_{\theta} \equiv \dot{\theta} f_{\phi}^{2}, \quad Y_{\theta} \equiv \frac{n_{\theta}}{s},
$$

and call $n_{\theta}$ a charge density and $Y_{\theta}$ a yield. Even though $Y_{\theta}$ is a redshift-invariant quantity, the evaluation of $Y_{\theta}$ from the initial condition is model dependent and is thoroughly discussed later in this Letter.

We will eventually consider the case where the axion originates from a phase direction of a complex scalar field $P$ whose vacuum expectation value spontaneously breaks a $U(1)$ global symmetry,

$$
P \equiv \frac{1}{\sqrt{2}}\left(S+f_{\phi}\right) e^{i \frac{\phi}{f_{\phi}}},
$$

where $S$ and $\phi$ are the radial and angular (axion) modes, respectively. However, the kinetic misalignment mechanism can be understood without referring to $P$. The nonzero axion velocity corresponds to a rotation of $P$.

We assume the potential of the axion is

$$
V=m_{\phi}(T)^{2} f_{\phi}^{2}\left(1-\cos \frac{\phi}{f_{\phi}}\right),
$$

where the axion mass $m_{\phi}(T)$ may depend on temperature $T$. If $Y_{\theta}$ is sufficiently small, axion field oscillations begin at $T_{*}$ where $m_{\phi}\left(T_{*}\right)=3 H\left(T_{*}\right)$, yielding the conventional misalignment mechanism.
Our key point is that, if the axion kinetic energy $K=$ $\dot{\theta}^{2} f_{\phi}^{2} / 2$ is larger than its potential energy $V(\phi)$ at the conventional oscillation temperature $T_{*}$, the axion simply overcomes the potential barrier and the misalignment angle continues to change at the rate $\dot{\theta}(T)$. This evolution ceases when the kinetic energy $K$ redshifts to the height of the potential barrier, $V_{\max }=2 m_{\phi}^{2}(T) f_{\phi}^{2}$, at the temperature we call $T^{\prime}$,

$$
\dot{\theta}\left(T^{\prime}\right)=2 m_{\phi}\left(T^{\prime}\right) .
$$

Subsequently, the axion is trapped by the potential barrier and oscillates around the minimum. The onset of oscillation is delayed if this trapping happens after the conventional oscillation temperature, $T^{\prime}<T_{*}$. Equivalently, kinetic misalignment is at play when

$$
m_{\phi}\left(T^{\prime}\right) \geq 3 H\left(T^{\prime}\right) .
$$

If the axion mass changes adiabatically, the number density is conserved. The energy density of the axion oscillation $\rho_{\phi}$ normalized by the entropy density reads

$$
\frac{\rho_{\phi}}{s}=m_{\phi}(0) \frac{n_{\phi}\left(T^{\prime}\right)}{s\left(T^{\prime}\right)}=C m_{\phi}(0) Y_{\theta} .
$$

The axion abundance only depends on $Y_{\theta}$ and the mass of the axion in the vacuum, and is independent of the evolution of the axion mass.

The analytic estimate thus far predicts $C=1$ since

$$
n_{\phi}\left(T^{\prime}\right)=\frac{V_{\phi}}{m_{\phi}\left(T^{\prime}\right)} \simeq 2 m_{\phi}\left(T^{\prime}\right) f_{\phi}^{2} \simeq \dot{\theta}\left(T^{\prime}\right) f_{\phi}^{2}=n_{\theta} .
$$

However, this estimate is not precise because $\rho_{\phi}$ is assumed to scale as $R^{-3}$ as soon as the oscillation starts at $T^{\prime}$. Since the oscillation starts near the top of the cosine potential where the potential gradient is small, there is a further delay in the oscillation and this anharmonicity enhances the axion abundance. This nonlinear effect calls for a numerical analysis, which we perform in the Supplemental Material [56] and determine $C \simeq 2$.

The kinetic misalignment mechanism is effective when

$$
Y_{\theta}>Y_{\text {crit }}=\frac{n_{\phi}\left(T_{*}\right)}{s\left(T_{*}\right)} \sim \frac{f_{\phi}^{2}}{M_{\mathrm{Pl}} T_{*}} .
$$

Furthermore, sufficient axion dark matter results if $m_{\phi}(0) Y_{\theta} \simeq T_{e}$, where $T_{e}$ is the temperature of matterradiation equality. The condition for kinetic misalignment in Eq. (8) then requires $m_{\phi}(0) f_{\phi}^{2} \lesssim T_{e} T_{*} M_{\mathrm{Pl}}$.

We now estimate $Y_{\text {crit }}$ for the QCD axion, $a$, and take $m_{a} \propto T^{-4}$ for $T>\Lambda_{\mathrm{QCD}}$ from the dilute instanton gas approximation (also see the lattice results in Refs. [62-66]), 


$$
Y_{\text {crit }}=0.11\left(\frac{f_{a}}{10^{9} \mathrm{GeV}}\right)^{\frac{13}{6}}
$$

For $Y_{\theta} \gg Y_{\text {crit }}$ the axion abundance is

$$
\Omega_{a} h^{2} \simeq \Omega_{\mathrm{DM}} h^{2}\left(\frac{10^{9} \mathrm{GeV}}{f_{a}}\right)\left(\frac{Y_{\theta}}{40}\right),
$$

which is independent of the axion mass evolution. For $f_{a} \gtrsim 1.5 \times 10^{11} \mathrm{GeV}$, kinetic misalignment cannot yield axion dark matter, since Eqs. (9) and (10) then give $Y_{\theta}<$ $Y_{\text {crit }}$ and the usual misalignment mechanism results.

The relevant numerical results and analytic derivations are thoroughly presented in the Supplemental Material [56].

Rotation from higher dimensional operators.Assuming that the potential of $|P|$ is sufficiently flat, a large field value may arise during inflation as an initial condition, by quantum fluctuations, or due to a negative Hubble-induced mass. For large enough initial field value $\left|P_{i}\right|$, the explicit breaking of the global symmetry by higher dimensional operators may become important. Such operators give a potential gradient to the angular direction of $P$ and drive angular motion. By the cosmic expansion, the field value $|P|$ decreases and the higher dimensional operator becomes ineffective. The angular direction then has a flat potential and $P$ rotates about the origin. This dynamics is the same as that in Affleck-Dine baryogenesis $[18,19]$ with supersymmetric partners of quarks and leptons.

The rotation is understood as a state with an asymmetry of the global charge. The density of the Noether charge associated with the symmetry $P \rightarrow e^{i \alpha} P$ is

$$
n_{\theta}=i P \dot{P}^{*}-i P^{*} \dot{P}
$$

which is nonzero for a rotating $P$ and reduces to Eq. (1) when $|P|$ is relaxed to $f_{\phi} / \sqrt{2}$.

At the onset of the rotation, the asymmetry is

$$
n_{\theta}=\epsilon \frac{V\left(P_{i}\right)}{m_{S}\left(P_{i}\right)}, \quad \epsilon \lesssim 1,
$$

where $\epsilon$, defined by this equation, parametrizes how close the trajectory is to a circular motion, which maximizes the asymmetry for a fixed energy. The size of $\epsilon$ is determined by the potential gradient of the angular direction relative to that of the radial direction.

Soon after the onset of the rotation, $n_{\theta} R^{3}$ becomes a conserved quantity, implying that $\dot{\theta} \propto R^{-3}|P|^{-2}$ redshifts slower for $|P| \gg f_{a}$ than if $|P|$ is fixed at $f_{\phi} / \sqrt{2}$. This slower redshift plays an important role and may explain why a $\dot{\theta}$ sufficiently large to affect the axion dynamics around the QCD phase transition has been neglected.
In what follows, we explicitly demonstrate the kinetic misalignment mechanism using a quartic and a quadratic potential.

Model with quartic potential.-We first demonstrate kinetic misalignment with the quartic potential for the global symmetry breaking field $P$,

$$
V=\lambda^{2}\left(|P|^{2}-\frac{f_{\phi}^{2}}{2}\right)^{2}, \quad \lambda^{2}=\frac{1}{2} \frac{m_{S}^{2}}{f_{\phi}^{2}},
$$

where $m_{S}$ is the vacuum mass of the radial degree of freedom $S$. Simply following the terminology in supersymmetric theories, we call $S$ the saxion. For small $\lambda$, the saxion has a flat potential and may obtain a large field value during inflation. At an initial field value $\left|P_{i}\right|=S_{i} / \sqrt{2}$, the saxion mass is $\sqrt{3} \lambda S_{i}$. The saxion begins to oscillate when the mass exceeds $3 H$. Assuming radiation domination, the temperature at which this occurs is

$$
T_{\text {osc }} \simeq 2 \times 10^{12} \mathrm{GeV}\left(\frac{S_{i}}{10^{17} \mathrm{GeV}}\right)^{\frac{1}{2}}\left(\frac{\lambda}{10^{-10}}\right)^{\frac{1}{2}} .
$$

When the oscillation starts, the asymmetry given in Eq. (12) is $n_{\theta}=\epsilon \lambda S_{i}^{3} /(4 \sqrt{3})$, corresponding to the yield

$$
Y_{\theta} \equiv \frac{n_{\theta}}{s} \simeq 40 \epsilon\left(\frac{S_{i}}{10^{17} \mathrm{GeV}}\right)^{\frac{3}{2}}\left(\frac{10^{-10}}{\lambda}\right)^{\frac{1}{2}},
$$

which remains constant unless entropy is later injected.

When $S \gg f_{\phi}$, the quartic term dominates and the energy density of the rotation redshifts as $R^{-4}, S \propto R^{-1}$, and $\dot{\theta} \propto R^{-1}$. When $S \simeq f_{\phi}$, the quadratic term dominates and the radial mode's energy density begins to redshift as $R^{-3}$ and $\dot{\theta}$ follows the usual scaling $R^{-3}$. Hence, a large initial $S$ slows down the redshift of $\dot{\theta}$.

We assume that $P$ is thermalized to avoid overclosure from the radial mode. As shown in Ref. [55], even after thermalization, $P$ continues to rotate because it is energetically favorable to keep the charge asymmetry in the rotation rather than particle excitations. At thermalization, an elliptical trajectory becomes circular and the charge density $n_{\theta}$ stays conserved up to cosmic expansion. From charge conservation and the scaling of $P$, one finds that the angular mode's energy density $\rho_{\theta}=n_{\theta}^{2} / S^{2}$ decreases as $R^{-4}\left(R^{-6}\right)$ for $S \gg f_{\phi}\left(S \simeq f_{\phi}\right)$. The angular mode does not dominate the energy density since $\rho_{\theta}$ never redshifts slower than radiation.

The radius $S$ eventually settles to $f_{\phi}$, and the axion rapidly moves along the bottom of the potential in Eq. (13). The kinetic misalignment mechanism determines the axion abundance if $Y_{\theta}>Y_{\text {crit }}$.

The available parameter space for QCD axion dark matter is summarized in Fig. 2, where various constraints are discussed in this and the next sections. The red region 


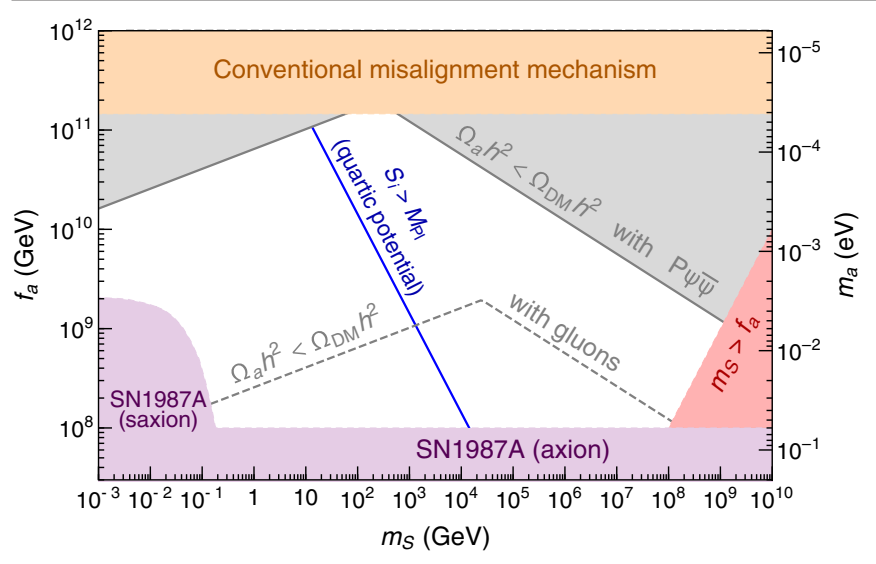

FIG. 2. The parameter space of the QCD axion decay constant $f_{a}$ (or mass $m_{a}$ ) and the saxion vacuum mass $m_{S}$ compatible with the observed dark matter abundance. The blue line excludes high $m_{S}$ for the quartic potential. Applicable to both quartic and quadratic potentials, the gray region is ruled out for a maximal thermalization rate, while the constraint is the gray dashed line for thermalization via gluons only.

violates unitarity of the saxion self-interaction, while the purple region is excluded since the duration of the neutrino emission in a supernova core is altered by the emission of axions [8-14] or saxions [67]. In the orange region, the conventional misalignment mechanism instead is operative since $Y_{\theta}<Y_{\text {crit }}$ from Eqs. (9) and (10). The axion abundance is enhanced for larger $S_{i}$, but $S_{i}$ cannot exceed the Planck scale, giving an upper bound on $f_{a}$ based on Eqs. (10) and (15)

$$
f_{a} \lesssim 10^{9} \mathrm{GeV} \epsilon^{2}\left(\frac{\mathrm{TeV}}{m_{S}}\right),
$$

corresponding to the blue line in Fig. 2 for $\epsilon=1$ and gets stronger if dilution due to entropy production is present.

The energy density of the saxion must be depleted to avoid cosmological disaster, e.g., excessive dark radiation from the decay to axions. The saxion can be thermalized by scattering with gluons and with fermions $\psi$ via a Yukawa coupling $y P \psi \bar{\psi}$. The interaction rate with the gluons and fermions is suppressed for larger $f_{a}$. The scattering with gluons (fermions) can successfully deplete the saxion in the region below the positively sloped segment of the gray dashed line (gray boundary) in Fig. 2. We present the rigorous examination of the thermalization constraints in the Supplemental Material [56]. A wide range of $f_{a} \lesssim$ $10^{11} \mathrm{GeV}$ is possible between such gray lines and the blue line from Eq. (16).

A sufficient amount of QCD axion dark matter requires that $m_{S}$ and hence the quartic coupling are small; namely the potential of $P$ is flat. This is because a late start of the oscillation of $P$ enhances the charge to entropy ratio.

Supersymmetric models. - The kinetic misalignment mechanism benefits from supersymmetry, where symmetry breaking fields naturally have flat potentials.
We consider the case where the saxion has a nearly quadratic potential with a typical mass $m_{S}$. This is the case for (1) a model with global symmetry breaking by dimensional transmutation due to the renormalization group running of the soft mass [68],

$$
V=m_{S}^{2}|P|^{2}\left(\ln \frac{2|P|^{2}}{f_{\phi}^{2}}-1\right)
$$

(2) a two-field model with soft masses,

$W=X\left(P \bar{P}-V_{P}^{2}\right), \quad V_{\text {soft }}=m_{P}^{2}|P|^{2}+m_{\bar{P}}^{2}|\bar{P}|^{2}$,

where $X$ is a chiral multiplet whose $F$ term fixes the global symmetry breaking fields $P$ and $\bar{P}$ along the moduli space $P \bar{P}=V_{P}^{2}$, and (3) global symmetry breaking by quantum corrections in gauge mediation [69-71].

For a nearly quadratic potential, the rotation of $P$ can occur in the same manner as the rotation of scalars in Affleck-Dine baryogenesis $[18,19]$. In the early Universe $P$ may obtain a negative mass term by a Planck scalesuppressed coupling to the total energy density,

$$
V=-c_{H} H^{2}|P|^{2},
$$

where $H$ is the Hubble scale and $c_{H}$ is an $\mathcal{O}(1)$ constant. For $H>m_{S}$, the saxion is driven to a large field value.

We consider explicit global symmetry breaking by a higher dimensional superpotential,

$$
W=\frac{P^{n+1}}{M^{n-2}} .
$$

The $F$-term potential from Eq. (20) stabilizes the saxion $S \equiv \sqrt{2}|P|$ against the negative Hubble induced mass. The saxion tracks the minimum of the potential $[19,72]$

$$
S(H) \simeq\left(H^{2} M^{2 n-4}\right)^{\frac{1}{2 n-2}} .
$$

Once $H$ drops below $m_{S}$, the saxion begins to oscillate. Meanwhile, the supersymmetry breaking $A$-term potential associated with Eq. (20)

$$
V \simeq(n+1) A \frac{P^{n+1}}{M^{n-2}}+\text { H.c. },
$$

breaks the global symmetry explicitly, inducing the rotation of $P$. Here $A$ is of order the gravitino mass in gravity mediation. According to Eq. (12), the asymmetry at the onset of the rotation is

$$
n_{\theta} \simeq A S\left(m_{S}\right)^{2},
$$

if the initial phase is not accidentally aligned with the minimum. At a large field value, the saxion mass tends to be 
dominated by the gravity mediated one, so $m_{S}=\mathcal{O}(A)$ and $n_{\theta}$ is of order $\rho_{P} / m_{S}$. The charge density normalized by the saxion energy density remains constant despite the cosmic expansion. [For $M=\mathcal{O}\left(M_{\mathrm{Pl}}\right)$ and $A=\mathcal{O}(\mathrm{TeV})$, a shift to the $C P$ violating phase of the strong interaction from the explicit PQ symmetry breaking is smaller than the experimental upper bound if $n>7-9$ for $f_{a}=10^{9}-10^{12} \mathrm{GeV}$ ].

Because of the large initial field value, the saxion tends to dominate the energy density of the Universe, which we assume hereafter. Regardless, $P$ has to be thermalized eventually. After thermalization completes at the temperature $T_{\text {th }}, P$ rotates with a vanishing ellipticity and with the total charge $n_{\theta} R^{3}=S^{2} \dot{\theta} R^{3}$ conserved. The charge conservation implies that $\dot{\theta}$ stays constant for $|P| \gg f_{\phi}$ before following the usual $R^{-3}$ scaling when $|P| \simeq f_{\phi}$. Thermalization transfers the energy of the radial motion of $P$ into radiation. The remaining energy is associated with a circular motion, $\rho_{\theta}$. The final yield is

$$
Y_{\theta}=\frac{n_{\theta}}{s}=\epsilon \frac{3 T_{\mathrm{th}}}{4 m_{S}}, \quad \epsilon \equiv \frac{n_{\theta}}{\frac{\rho_{P}}{m_{S}}-n_{\theta}} \simeq \frac{A}{m_{S}},
$$

and $\epsilon \lesssim 1$ measures the amount of angular rotations relative to radial oscillations.

After thermalization, the equation of motion fixes $\dot{\theta}=m_{S}$, with which one can easily show by conservation of energy and $U(1)$ charge that $|P|$ does not immediately drop to $f_{\phi}$ as usual thermalization does for a scalar without a $U(1)$ charge. Instead, $|P|$ redshifts by the cosmic expansion. The energy density of the circular rotation decreases as $R^{-3}\left(R^{-6}\right)$ for $|P| \gg f_{\phi}\left(|P| \simeq f_{\phi}\right)$. Right after thermalization, the Universe is still dominated by the circular rotation, but after the $R^{-6}$ scaling begins, the Universe is eventually dominated by the thermal bath created by the aforementioned thermalization process.

We focus on the QCD axion and discuss whether sufficient axion dark matter can be produced. From Eqs. (10) and (24), the thermalization temperature needed to obtain the observed dark matter abundance is

$$
T_{\mathrm{th}} \simeq 50 \frac{m_{S}}{\epsilon}\left(\frac{f_{a}}{10^{9} \mathrm{GeV}}\right)
$$

Since $T_{\text {th }} \gg m_{S}$, thermal dissipation is necessary. To obtain the dark matter abundance, this thermalization temperature must be above or equal to that in Eq. (25). (In the former case, the correct abundance can be obtained without matter domination by $P$ or extra dilution.) This leads to an upper bound on $f_{a}$, as shown by the negatively sloped gray dashed line in Fig. 2 for $\epsilon=1$ for thermalization by scattering with gluons. A wider range of $f_{a} \lesssim 10^{11} \mathrm{GeV}$ becomes possible for fermion $\psi$ scatterings (shown by the negatively sloped gray boundary) compared to gluon scatterings. A detailed discussion is provided in the
Supplemental Material [56]. In supersymmetric models, larger values of $m_{S}$ become viable compared to the case of the quartic potential (blue line).

Discussion.-We presented the kinetic misalignment mechanism, where the dark matter abundance of a generic axionlike particle is determined by the initial field velocity, as opposed to the conventionally assumed initial misalignment. We then showed that this can yield QCD axion dark matter for any $f_{a}$ below $1.5 \times 10^{11} \mathrm{GeV}$, down to the minimum value allowed by supernovae constraints, $f_{a} \sim 10^{8} \mathrm{GeV}$. We studied in detail the full cosmological evolution of the PQ field in both quartic and quadratic potentials, with the results in Fig. 2 showing that kinetic misalignment is successful over a wide range of parameters. Besides signals in axion searches in the mass range $\mathcal{O}(0.1-100) \mathrm{meV}$, kinetic misalignment can provide a unified origin of dark matter and the cosmological excess of matter over antimatter [55].

Throughout the Letter, we assumed the field rotation remains coherent. If the radial direction of the global symmetry breaking field has a potential flatter than quadratic, an instability develops and the coherent rotation fragments into inhomogeneous configurations [73-76]. In this case, it is important to investigate how the inhomogeneity impacts the axion abundance.

The work was supported in part by the DOE Early Career Grant DE-SC0019225 (R. C.), the DOE Grants No. DE-AC02-05CH11231 (L.H.) and No. DESC0009988 (K. H.), the NSF Grant No. NSF-1638509 (L. H.), as well as the Raymond and Beverly Sackler Foundation Fund (K. H.).

[1] G. 't Hooft, Phys. Rev. Lett. 37, 8 (1976).

[2] R. J. Crewther, P. Di Vecchia, G. Veneziano, and E. Witten, Phys. Lett. 88B, 123 (1979); 91B, 487 (1980).

[3] C. A. Baker et al., Phys. Rev. Lett. 97, 131801 (2006).

[4] R. D. Peccei and H. R. Quinn, Phys. Rev. Lett. 38, 1440 (1977).

[5] R. D. Peccei and H. R. Quinn, Phys. Rev. D 16, 1791 (1977).

[6] S. Weinberg, Phys. Rev. Lett. 40, 223 (1978).

[7] F. Wilczek, Phys. Rev. Lett. 40, 279 (1978).

[8] J. R. Ellis and K. A. Olive, Phys. Lett. B 193, 525 (1987).

[9] G. Raffelt and D. Seckel, Phys. Rev. Lett. 60, 1793 (1988).

[10] M. S. Turner, Phys. Rev. Lett. 60, 1797 (1988).

[11] R. Mayle, J. R. Wilson, J. R. Ellis, K. A. Olive, D. N. Schramm, and G. Steigman, Phys. Lett. B 203, 188 (1988).

[12] G. G. Raffelt, Lect. Notes Phys. 741, 51 (2008).

[13] J. H. Chang, R. Essig, and S. D. McDermott, J. High Energy Phys. 09 (2018) 051.

[14] P. Carenza, T. Fischer, M. Giannotti, G. Guo, G. Martinez-Pinedo, and A. Mirizzi, J. High Energy Phys. 10 (2019) 016.

[15] J. Preskill, M. B. Wise, and F. Wilczek, Phys. Lett. 120B, 127 (1983). 
[16] L. F. Abbott and P. Sikivie, Phys. Lett. 120B, 133 (1983).

[17] M. Dine and W. Fischler, Phys. Lett. 120B, 137 (1983).

[18] I. Affleck and M. Dine, Nucl. Phys. B249, 361 (1985).

[19] M. Dine, L. Randall, and S. D. Thomas, Nucl. Phys. B458, 291 (1996).

[20] R. Holman, S. D. H. Hsu, T. W. Kephart, E. W. Kolb, R. Watkins, and L. M. Widrow, Phys. Lett. B 282, 132 (1992).

[21] S. M. Barr and D. Seckel, Phys. Rev. D 46, 539 (1992).

[22] M. Kamionkowski and J. March-Russell, Phys. Lett. B 282, 137 (1992).

[23] M. Dine, arXiv:hep-th/9207045.

[24] J. K. Vogel et al., arXiv:1302.3273.

[25] E. Armengaud et al., J. Instrum. 9, T05002 (2014).

[26] A. Arvanitaki and A. A. Geraci, Phys. Rev. Lett. 113, 161801 (2014).

[27] G. Rybka, A. Wagner, K. Patel, R. Percival, K. Ramos, and A. Brill, Phys. Rev. D 91, 011701(R) (2015).

[28] P. Sikivie, Phys. Rev. Lett. 113, 201301 (2014).

[29] A. Caldwell, G. Dvali, B. Majorovits, A. Millar, G. Raffelt, J. Redondo, O. Reimann, F. Simon, and F. Steffen (MADMAX Working Group), Phys. Rev. Lett. 118, 091801 (2017).

[30] B. T. McAllister, G. Flower, E. N. Ivanov, M. Goryachev, J. Bourhill, and M.E. Tobar, Phys. Dark Universe 18, 67 (2017).

[31] V. Anastassopoulos et al. (TASTE Collaboration), J. Instrum. 12, P11019 (2017).

[32] A. Arvanitaki, S. Dimopoulos, and K. Van Tilburg, Phys. Rev. X 8, 041001 (2018).

[33] A. A. Geraci et al. (ARIADNE Collaboration), Springer Proc. Phys. 211, 151 (2018).

[34] M. Baryakhtar, J. Huang, and R. Lasenby, Phys. Rev. D 98, 035006 (2018).

[35] N. Du et al. (ADMX Collaboration), Phys. Rev. Lett. 120, 151301 (2018).

[36] D. J. E. Marsh, K.-C. Fong, E. W. Lentz, L. Smejkal, and M. N. Ali, Phys. Rev. Lett. 123, 121601 (2019).

[37] M. Lawson, A. J. Millar, M. Pancaldi, E. Vitagliano, and F. Wilczek, Phys. Rev. Lett. 123, 141802 (2019).

[38] M. Zarei, S. Shakeri, M. Abdi, D. J. E. Marsh, and S. Matarrese, arXiv:1910.09973.

[39] R. T. Co, L. J. Hall, and K. Harigaya, Phys. Rev. Lett. 120, 211602 (2018).

[40] K. Harigaya and J. M. Leedom, J. High Energy Phys. 06 (2020) 034.

[41] M. S. Turner, Phys. Rev. D 33, 889 (1986).

[42] D. H. Lyth, Phys. Rev. D 45, 3394 (1992).

[43] L. Visinelli and P. Gondolo, Phys. Rev. D 80, 035024 (2009).

[44] R. T. Co, E. Gonzalez, and K. Harigaya, J. High Energy Phys. 05 (2019) 163.

[45] F. Takahashi and W. Yin, J. High Energy Phys. 10 (2019) 120.

[46] P. Sikivie, Phys. Rev. Lett. 48, 1156 (1982).
[47] S. Chang, C. Hagmann, and P. Sikivie, Phys. Rev. D 59, 023505 (1998).

[48] T. Hiramatsu, M. Kawasaki, and K. Saikawa, J. Cosmol. Astropart. Phys. 08 (2011) 030.

[49] T. Hiramatsu, M. Kawasaki, K. Saikawa, and T. Sekiguchi, J. Cosmol. Astropart. Phys. 01 (2013) 001.

[50] M. Kawasaki, K. Saikawa, and T. Sekiguchi, Phys. Rev. D 91, 065014 (2015).

[51] A. Ringwald and K. Saikawa, Phys. Rev. D 93, 085031 (2016); 94, 049908(A) (2016).

[52] K. Harigaya and M. Kawasaki, Phys. Lett. B 782, 1 (2018).

[53] A. Caputo and M. Reig, Phys. Rev. D 100, 063530 (2019).

[54] L. Visinelli and P. Gondolo, Phys. Rev. D 81, 063508 (2010).

[55] R. T. Co and K. Harigaya, Phys. Rev. Lett. 124, 111602 (2020).

[56] See Supplemental Material at http://link.aps.org/ supplemental/10.1103/PhysRevLett.124.251802, which includes Refs. [57-61], for the review of the conventional misalignment mechanism, a numerical study of the kinetic misalignment mechanism, and the analysis of the thermalization of the saxion.

[57] J. Yokoyama, Phys. Rev. D 70, 103511 (2004).

[58] M. Bastero-Gil, A. Berera, and R. O. Ramos, J. Cosmol. Astropart. Phys. 09 (2011) 033.

[59] M. Drewes, arXiv:1012.5380.

[60] K. Mukaida and K. Nakayama, J. Cosmol. Astropart. Phys. 01 (2013) 017.

[61] K. Mukaida and K. Nakayama, J. Cosmol. Astropart. Phys. 03 (2013) 002.

[62] P. Petreczky, H.-P. Schadler, and S. Sharma, Phys. Lett. B 762, 498 (2016).

[63] S. Borsanyi et al., Nature (London) 539, 69 (2016).

[64] F. Burger, E.-M. Ilgenfritz, M. P. Lombardo, and A. Trunin, Phys. Rev. D 98, 094501 (2018).

[65] C. Bonati, M. D’Elia, G. Martinelli, F. Negro, F. Sanfilippo, and A. Todaro, J. High Energy Phys. 11 (2018) 170.

[66] M. Gorghetto and G. Villadoro, J. High Energy Phys. 03 (2019) 033.

[67] N. Ishizuka and M. Yoshimura, Prog. Theor. Phys. 84, 233 (1990).

[68] P. Moxhay and K. Yamamoto, Phys. Lett. 151B, 363 (1985).

[69] N. Arkani-Hamed, G. F. Giudice, M. A. Luty, and R. Rattazzi, Phys. Rev. D 58, 115005 (1998).

[70] T. Asaka and M. Yamaguchi, Phys. Lett. B 437, 51 (1998).

[71] T. Asaka and M. Yamaguchi, Phys. Rev. D 59, 125003 (1999).

[72] K. Harigaya, M. Ibe, M. Kawasaki, and T. T. Yanagida, J. Cosmol. Astropart. Phys. 11 (2015) 003.

[73] A. Kusenko and M. E. Shaposhnikov, Phys. Lett. B 418, 46 (1998).

[74] K. Enqvist and J. McDonald, Phys. Lett. B 425, 309 (1998).

[75] K. Enqvist and J. McDonald, Nucl. Phys. B538, 321 (1999).

[76] S. Kasuya and M. Kawasaki, Phys. Rev. D 61, 041301(R) (2000). 